\title{
Croissance urbaine et durée des épisodes migratoires : l'exemple de Genève au 19ème siècle
}

\author{
ADRIEN REMUND ${ }^{1}$
}

\begin{abstract}
Résumé
Dans cette étude, nous montrons comment la croissance urbaine est directement liée à la durée moyenne des séjours en ville. Alors que dans la narration classique de l'exode rural, l'urbanisation n'est présentée que comme la conséquence de la migration massive de la population rurale vers la ville, cette affirmation a été fortement nuancée par des travaux d'historiens plus récents. Afin de montrer empiriquement comment la durée moyenne des épisodes migratoires influence la croissance urbaine, nous appliquons des méthodes inspirées de l'épidémiologie au cas de Genève au 19ème siècle. Cette analyse dépeint une évolution irrégulière du temps passé en ville, cadencée par des phases de forte mobilité et de stabilisation. Ces dernières coïncident avec les périodes de forte croissance de la population genevoise.
\end{abstract}

Mots-clés

Croissance urbaine, migrations temporaires, exode rural, Genève, 19ème siècle.

\section{Summary}

In this study, we show how urban growth is directly linked to the mean duration of stay in the city. In the classical narrative of rural flight, urbanisation is pictured as the direct consequence of mass migration of rural dwellers to the city. This proposition has however been restated by the more recent works of historians. In order to empirically show how the mean duration of migration spells influences urban growth, we apply epidemiological methods to the case of nineteenth-century Geneva. This analysis pictures an irregular evolution of the time spent in town, marked by successive phases of high mobility and stabilisation. The latter coincide with the periods of rapid growth of the town's population.

\section{Keywords}

Urban growth, temporary migration, rural flight, Geneva, 19th century.

1. Institut d'études démographiques et du parcours de vie (I-Demo), Université de Genève. 


\section{Introduction}

Le 19 ème siècle a été le témoin d'au moins deux tournants majeurs dans l'histoire démographique de l'Europe. Premièrement, la population du continent a progressivement quitté un monde malthusien caractérisé par une mortalité et une fécondité élevées, pour un nouveau régime marqué par une mortalité et une fécondité basses. Le décalage temporel entre la baisse de la mortalité et celle de la fécondité au cours de la transition démographique a généré une croissance de la population inédite, ce que Chesnais appelle «le multiplicateur transitionnel de population» (1979). Certes plus modérée en Europe qu'ailleurs, cette croissance démographique a toutefois signifié un doublement de la population initiale en moyenne (Chesnais, 1979).

Un second aspect central de l'histoire du 19ème siècle est sans conteste l'urbanisation. En un siècle, la part des citadins dans la population du continent passe d'un dixième à un tiers (Bairoch, 1997 ; Pinol, 2003). Parce que la sortie du régime malthusien s'est effectuée grâce à l'industrialisation, la localisation de la production économique a été transférée des campagnes vers les villes. Dès lors, «si les progrès de l'agriculture permettent de nourrir un plus grand nombre de citadins, c'est l'industrie qui fournit l'essentiel des emplois urbains nouveaux» (Bairoch, 1997, p. 197). Ce basculement de l'activité économique engendre une attraction plus grande des villes sur la population grandissante des campagnes.

L'apparente simultanéité de migrations massives vers les villes et d'une croissance rapide de ces dernières a donné naissance au schéma historique dominant de l'exode rural. Sous ce paradigme, la croissance urbaine est présentée comme le corollaire direct de la migration urbaine. L'expansion des villes d'Europe serait donc simplement due au transfert massif et définitif des populations campagnardes vers les centres urbains, seule forme significative de migration dans un monde autrement statique. Ce modèle s'est appuyé sur le paradigme de la modernisation, qui a longtemps dominé l'interprétation de l'évolution démographique en général, et qui s'est matérialisée dans le postulat de la transition de la mobilité. Selon ce principe, les sociétés occidentales seraient passées d'une condition relativement figée marquée par une mobilité physique et sociale sévèrement limitée, vers des taux de mobilité toujours plus élevés (Zelinsky, 1971). Cependant, cette théorie n’a jamais passé l'écueil de la vérification empirique. Au contraire, les historiens ont peu à peu démontré la nature relativement mobile des sociétés d'Ancien Régime. Ce fut d'abord l'importance du life cycle service pour les jeunes 
adultes qui fut mise en avant (Laslett, 1977). Puis, grâce à la mesure du taux de migration dans l'Allemagne du 19ème siècle, on dut se faire à l'évidence que le niveau de mobilité dans les années 1830 était déjà aussi élevé qu'à la fin du 20ème siècle (Hochstadt, 1999).

Aujourd'hui, cette interprétation est définitivement taillée en brèche par la multiplication des études qui montrent que non seulement les migrations à l'intérieur des campagnes restent importantes, voire majoritaires, tout au long du 19ème siècle (Rosental, 1999), mais aussi que l'implantation durable en ville reste longtemps le fait d'une minorité (Faron, 1997 ; Hatt-Diener, 2004 ; Hochstadt, 1999 ; Lorenceau, 2001).

Ces nouvelles preuves de l'importance précoce des migrations dans l'histoire des populations européennes et la réaffirmation de leur diversité de formes et de fonctions impliquent que le volume des échanges entre les campagnes et les villes n'a pas été le seul moteur de la croissance urbaine. Plusieurs auteurs ont ainsi suggéré que la durée d'installation en ville pourrait être en partie responsable de leur croissance. Cette idée se retrouve notamment chez Rosental qui affirme qu'une "partie de l'augmentation de la population urbaine pourrait être simplement le résultat des migrants restant plus longtemps dans les villes» (Rosental, 2006, p. 470). Cette hypothèse est appuyée par l'exemple de Bâle, dont «le taux de croissance annuel (...) n'est pas corrélé au nombre de migrants qui arrivent chaque année» (Lorenceau, 2001, p. 93). Cette hypothèse de l'effet modérateur, voire proéminent, de la stabilisation des migrants sur la croissance urbaine n'a malheureusement été que rarement testée empiriquement. Cette étude vise à combler en partie cette lacune en contournant la difficulté majeure que représente la compilation des sources de données.

Toute étude historique proposant de comparer l'afflux vers la ville avec le reflux ou le temps passé dans celles-ci se heurte au fait que le premier phénomène est bien plus facile à mesurer que le second, particulièrement sur de longues périodes. En effet, le simple dénombrement des registres de population ou des permis de séjour offre rapidement une vue d'ensemble du nombre d'individus qui entrent en ville, alors que la durée des épisodes migratoires est une variable moins souvent disponible. Même lorsqu'elle figure dans les sources, par exemple sous la forme d'une date d'arrivée et d'une date de départ, son enregistrement nécessite le dépouillement de toutes les entrées une par une afin de calculer une durée moyenne de séjour. C'est pourtant cette méthode directe que nous tenterons d'appliquer dans un premier temps, en compilant les études déjà existantes. Face aux limites de cet exercice, nous envisagerons dans un second temps la possibilité d'évaluer cette durée 
moyenne des séjours par le biais de méthodes indirectes d'estimation inspirées de l'épidémiologie.

Ces deux analyses auront pour cadre le canton suisse de Genève ${ }^{2}$ sur l'ensemble du 19ème siècle. La situation de Genève à cette époque est celle d'une cité d'essence médiévale marquée par une isolation ancestrale vis-à-vis de ses voisins, et qui cherche à préserver son identité calviniste mise en péril par les changements économiques, démographiques et politique de l'époque. Bien qu'absente des grands bouleversements industriels que vivent à la même époque les bassins sidérurgiques d'Europe, la cité de Calvin ressent les effets du changement des temps de par la dépendance économique qu'elle entretient envers ses puissants voisins. Genève a été soumise très tôt à des flux migratoires importants. Précurseur du contrôle de la fécondité (Henry, Sauvy, 1956) et connaissant une croissance naturelle quasiment nulle dans la première moitié du 19ème siècle (Perrenoud, 1979; Schumacher, 2010), elle a été rapidement forcée de s'ouvrir à un apport externe en main d'œuvre qui longtemps n'a été que saisonnier, notamment en raison de la méfiance de la «Rome protestante» envers les puissances catholiques qui l'entouraient (Oris, Perroux, 2007). Jusqu'au milieu du 19ème siècle, Genève a donc expérimenté simultanément une forte intensité migratoire et une faible croissance urbaine. Puis, avec la destruction des fortifications qui débute en 1851, la ville est entrée dans une phase de croissance accélérée, essentiellement due au solde migratoire (Figure 1). Dans cette nouvelle dynamique, les années 1850-1860 et 1895-1914 sortent du lot comme étant marquées par un rythme de croissance particulièrement élevé. Cette succession de phases de stagnation et de croissance fait de Genève un cas particulièrement intéressant pour l'étude des relations entre migration et croissance urbaine.

2. Pour des raisons de disponibilité des données sur le nombre de permis de séjours, l'échelle d'analyse sera ici celle du canton. Celui-ci est cependant dominé de manière écrasante par la ville de Genève et sa banlieue immédiate (les communes des EauxVives, de Plainpalais et du Petit-Saconnex, qui sont rattachées à la ville de Genève en 1930), qui représentent ensemble $73 \%$ de la population cantonale en 1900 et $84 \%$ de la croissance démographique du canton entre 1822 et 1900. En d'autres termes, étudier la croissance du canton revient pratiquement à étudier celle de l'agglomération urbaine. 
Figure 1 Population du canton de Genève (1814-1914) 3

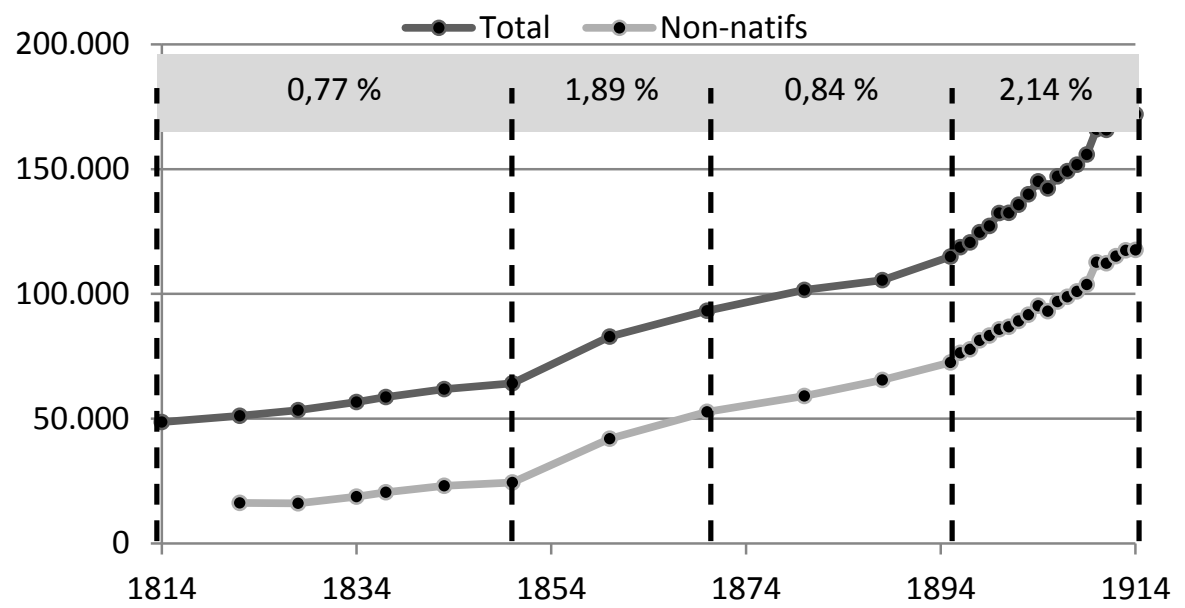

Source : Bairoch, Bovée, 1986, pp. 13-30.

\section{Sources}

À Genève, les registres des permis relatifs aux étrangers sont tenus depuis la fin du 18ème siècle, mais les séries ne sont continues que depuis 1816 (Engeli, Marin, 1974). Ils se présentent sous la forme de volumes dans lesquels chaque ligne contient les informations relatives à un migrant : nom, prénom, âge, origine, état civil, profession, nombre de renouvellements de permis, adresses occupées en villes et destination après Genève. La qualité de couverture de ces permis est garantie par l'efficacité du contrôle policier et social, omniprésent à Genève. En effet, la Chambre des Étrangers puis, dès 1844, la Police des Étrangers, ne délivre de permis que sur présentation des certificats d'origine et de bonne conduite du lieu d'habitation précédent, ainsi que la preuve d'une source de revenus à Genève. Tous les nouveaux arrivants sont informés de leur obligation de régularisation, notamment par les aubergistes, et les contrevenants ainsi que ceux qui les auraient hébergés risquent de lourdes sanctions financières, voire la prison (Schumacher, 2010). Les permis de séjour s'appliquent tant aux étrangers qu'aux Confédérés, exception faite des mouvements internes à Genève qui ne contribuent de toute façon pas à la croissance démographique du canton.

3. En gris, au-dessus du graphique, le taux d'accroissement annuel moyen sur la période pour la population totale. 
En pratique, il a existé au cours de l'histoire plusieurs types de permis ${ }^{4}$ (de séjour, de domicile, d'établissement, livrets de domestiques) permettant des séjours plus ou moins longs, et qui ont parfois coexisté. L'expérience montre que lorsque deux types de permis coexistent, celui qui permet une durée d'établissement plus longue ne représente qu'une proportion minime du total des permis. Par exemple, entre 1825 et 1843 , les permis de domicile ne représentent que 3,5\% du total des permis. Ce fait s'explique non-seulement par leur coût plus important, mais également par le fait que les non-Suisses sont exclus des permis de long terme (domicile ou établissement) jusqu'en 1879. L'étude du nombre des nouveaux permis délivrés par année (tous types confondus) permet de se faire une idée précise du nombre de personnes qui arrivent à Genève. Le total annuel de tous les permis, sauf les livrets de domestiques, est retranscrit dans les Comptes-rendus de l'administration du Conseil d'État (AEG BA 9), ce qui permet de retracer l'évolution du flux d'immigration annuel. Quant aux domestiques, on peut connaitre le nombre de livrets délivrés en comptant simplement le nombre d'entrées dans les registres annuels (AEG Étrangers F6-13). Le total des arrivées à Genève est donc représenté par la somme de ces deux relevés.

La connaissance de la population totale de la ville repose sur les recensements d'abord cantonaux de 1816, 1822, 1828, 1834, 1837 et 1843, puis fédéraux de 1850, 1860, 1870, 1880, 1888 et 1900. Plusieurs signes tendent à confirmer la qualité des premiers. Le déroulement sur deux à trois jours maximum, le nombre important d'agents recenseurs (jusqu'à 500 pour le recensement de 1843), la qualité d'instruction de ces agents, la périodicité de six ans et l'intérêt du gouvernement sont autant d'indications qui rassurent sur la qualité du recensement. Des études ont d'ailleurs exploité avec succès la qualité des inscriptions nominatives afin de retracer le parcours des individus d'un recensement à l'autre (Oris, Ritschard, 2007). Lors de ces premiers recensements, toute la population est comptée à l'exception des clients des auberges et des soldats. Elle inclut notamment les ouvriers étrangers, les voyageurs et les domestiques, que le gouvernement genevois cherche d'ailleurs à déduire de la population déclarée à la Confédération pour le calcul des contingents militaires (Schumacher, 2010).

Quant aux recensements fédéraux, ils répondent aux recommandations des congrès internationaux de statistique organisés dès 1853 sous l'impulsion d'Adolphe Quetelet (Schumacher, 2010). Ils reposent sur des

4. Nous utiliserons à l'avenir le terme de «permis» pour tout type de document donnant le droit de séjourner à Genève. 
bulletins de ménage remplis par les chefs de ménage eux-mêmes, inaugurant une méthode de collecte qui a survécu en Suisse jusqu'au recensement de 2000. L'Office fédéral de la statistique, créé pour l'occasion, juge que le niveau d'éducation de la population plus élevé que dans d'autres pays d'Europe occidentale garantit la qualité des réponses (Schumacher, 2010). Les statistiques du nombre d'habitants par origine proviennent des tables publiées par Bairoch et Bovée, se basant ellesmêmes sur l'Annuaire Statistique de Genève qui fait référence à la population résidante qui inclut les détenteurs de permis (Bairoch, Bovée, 1986).

\section{Volume et stabilité de l'immigration}

L'étude des migrations à Genève peut s'appuyer sur un certain nombre de données connues. Le nombre de permis accordés chaque année permet d'évaluer l'évolution du volume de l'immigration (Figure 2) ${ }^{5}$. De manière générale, on note une nette hausse des effectifs dans la seconde moitié du siècle qui concerne presque toutes les origines, à l'exception des Allemands, dont les effectifs stagnent jusqu'aux années 1890. Entre 1830 et 1900 , le nombre de permis octroyés est ainsi multiplié par six pour les Confédérés, par dix pour les Français et même par 250 pour les Italiens. L'annexion de la Savoie par la France et l'Unification italienne, par les modifications de regroupement qu'elles impliquent sur les origines des immigrants, n'expliquent qu'en partie ces évolutions extrêmes. En effet, si l'augmentation du nombre de Français dans les années 1880 reflète la disparition de la catégorie des Sardes ${ }^{6}$, le nombre d'Italiens n'explose véritablement que dans les années 1890 , soit bien après la fin de l'Unification.

5. Les origines ne sont pas indiquées pour les domestiques car seul le total des livrets de domestiques a été relevé. Avant la généralisation des livrets de domestiques en 1844 , il n'est pas possible de connaitre le nombre de domestiques arrivant en ville. Les valeurs de 1830 à 1844 sont donc extrapolées sur la base du ratio entre le nombre de livrets de domestiques et les autres permis sur la période 1844-1850. De plus, le total des permis subit des pics artificiels en 1844 et 1866 en raison du report d'anciennes permissions. Ces biais sont corrigés par la suite dans le calcul de la durée de séjour ( $c f$. note 10).

6. Pour une raison apparemment inconnue, l'administration genevoise continue de distinguer les citoyens anciennement sardes des citoyens français et italiens jusqu'en 1882. 
FIgURE 2 Permis accordés par origine (1830-1900)

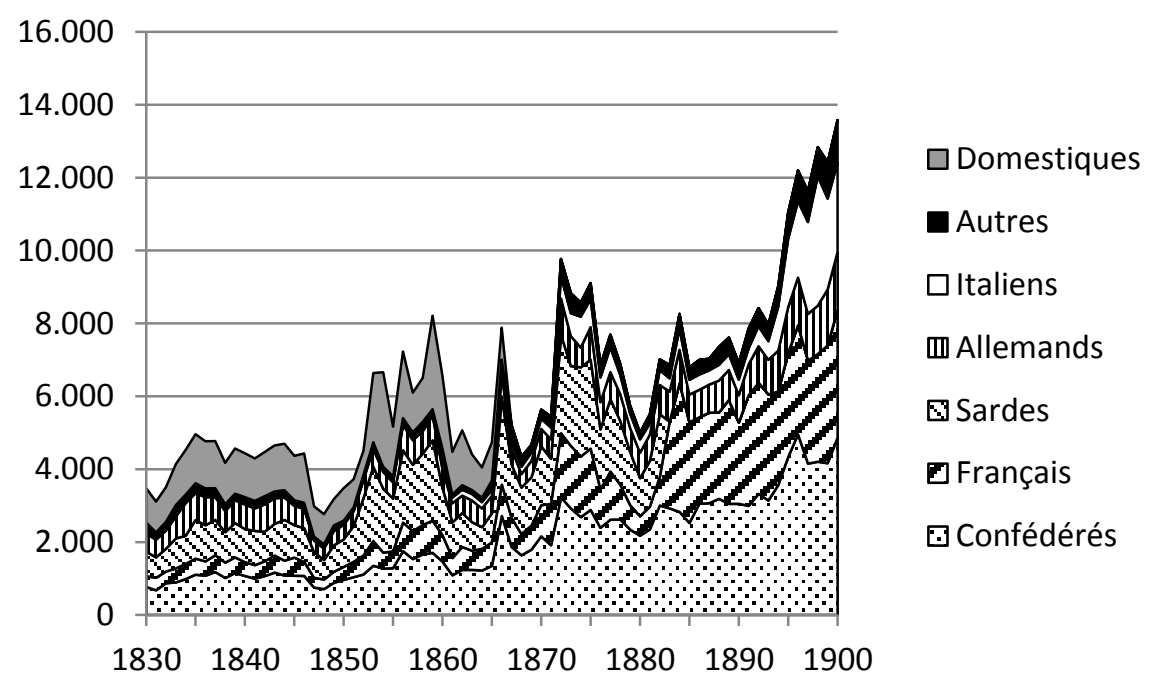

Source : AEG BA 9 ; AEG Étrangers F6-13 ; Schumacher, 2010, p. 230.

Le volume des arrivées n'est donc qu'en partie corrélé au rythme de la croissance urbaine. Certes, tant le nombre d'immigrants que la population augmentent fortement au cours du siècle. On ne peut pas nier non plus que l'explosion du nombre de permis après 1890 se reflète nettement sur l'accroissement démographique de cette période. En revanche, la hausse marquée des effectifs dans les années 1870 n'a qu'une incidence limitée sur la population du canton. La comparaison avec les années 1850 est particulièrement parlante. Ces deux décennies sont toutes deux marquées par une hausse du volume de l'immigration. Mais, alors que la seconde vague est près de deux fois plus importante que la première, elle n'engendre une croissance urbaine que moitié moindre.

En l'absence de séries fiables sur les sorties du canton de Genève, il est pratiquement impossible d'évaluer précisément l'importance de l'émigration (Schumacher, 2010). Les départs des citoyens genevois ne sont pas enregistrés et ceux des immigrés n'ont pas fait l'objet de comptesrendus tels qu'ils sont disponibles pour les arrivées. On possède toutefois une connaissance partielle du degré de stabilité des migrants grâce au dépouillement individuel des permis.

Plusieurs études ont utilisé les registres de permis pour des analyses ponctuelles de la durée de séjour à Genève. Elles ont en commun d'indiquer la proportion d'immigrés toujours présents après une année. Cet indicateur, si on l'observe sur la longue durée, montre des signes clairs 
d'augmentation au cours du temps (Figure 3). Cette hausse suggère que la durée moyenne des séjours effectués à Genève a progressé de manière générale durant le 19ème siècle. Bien qu'il ne s'agisse pas nécessairement d'un signe de disparition des migrations temporaires, ces premières indications révèlent une augmentation de la part des individus se fixant à Genève sur une plus longue période. D'environ un quart à la fin du 18ème siècle, la proportion d'immigrés restant plus d'une année atteint 50 \% à la fin du 19ème siècle, et même trois quarts à la veille de la Première Guerre mondiale.

Ainsi, indépendamment de l'augmentation du nombre de personnes qui arrivent à Genève chaque année, le temps qu'ils y passent semble avoir évolué dans un sens favorable à la croissance urbaine. Il reste toutefois encore à déterminer l'évolution plus fine et l'importance respective de ces deux composantes, ce qui n'est possible qu'en développant une méthode d'estimation indirecte couvrant, elle, l'ensemble du 19ème siècle.

FIgURE 3 Proportion d'immigrants toujours présents après une année (1790-1910)

hommes $\diamond$ femmes tous

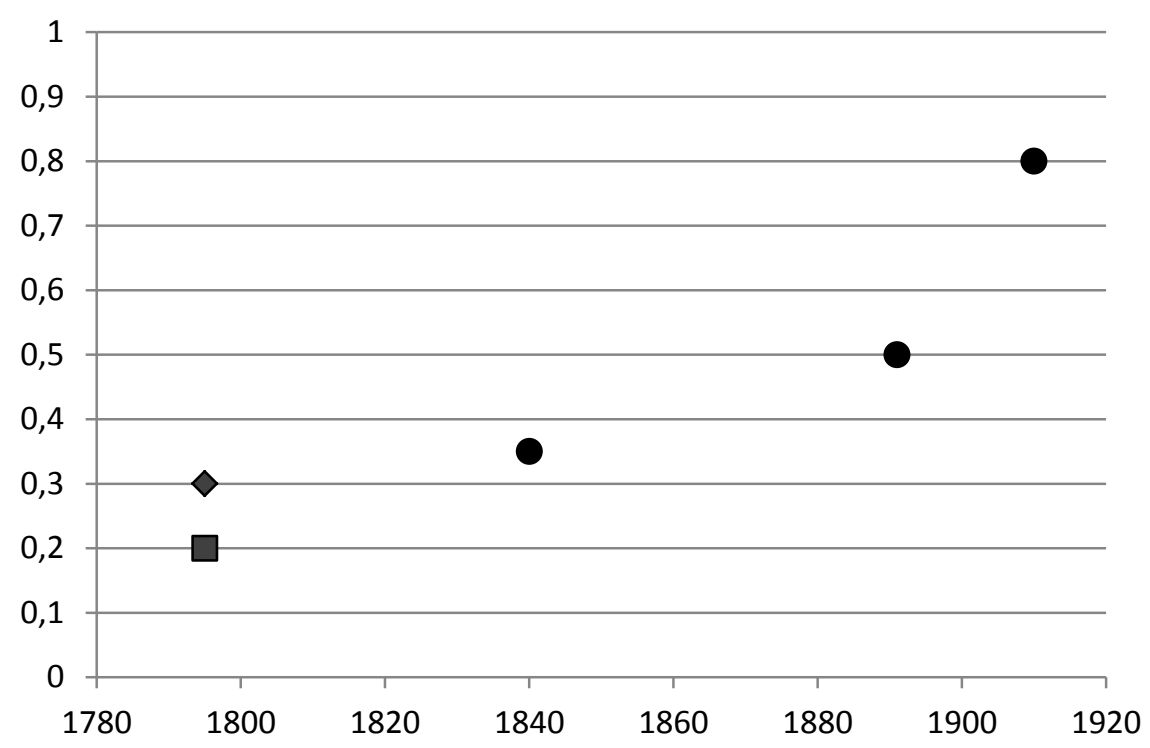

Sources : Engeli, Marin, 1974 ; Gille, 2009 ; Magnenat-Luthy, 1988 ; Remund, 2009. 


\section{Estimation indirecte du temps de séjour}

La question de l'estimation indirecte de la durée moyenne des séjours peut être abordée de manière détournée par l'angle de l'épidémiologie. Pour cela, il est nécessaire de définir certaines analogies qui peuvent à première vue paraitre surprenantes en mettant en avant les similitudes entre le temps passé dans une ville et la durée d'une infection.

Comme les analyses précédentes l'ont clairement montré, un modèle de croissance urbaine ne peut se limiter à enregistrer le nombre de personnes qui arrivent en ville, mais doit également prendre en compte le temps que les personnes y restent. De même, pour estimer la prévalence d'une maladie, connaitre son incidence ne suffit pas ; il faut également mesure le temps moyen d'infection. Par analogie avec un statut de santé (malade/non-malade), on peut donc observer la prévalence du statut résidentiel des non-natifs de la ville (présent/absent). Ce statut de présence est successivement acquis au moment de l'entrée en ville, puis perdu au moment du départ de celle-ci.

Ainsi, en mettant à profit cette similarité, il est possible d'étudier les mouvements dans et hors de la ville de la même manière que s'il s'agissait d'infections et de guérisons. Dans ce schéma, la présence des immigrés à un moment donné en ville peut être comparée à la prévalence de la migration en ville. Le nombre de nouveaux arrivants pendant une certaine période peut être défini comme l'incidence de la migration. Enfin, la durée moyenne de séjour en ville se compare dans ce modèle épidémiologique à la durée moyenne d'infection.

Ces trois mesures sont habituellement définies de la manière suivante dans l'étude de la dynamique d'une maladie nouvelle (Preston et al., 2000, p. 91)7. L'incidence d'une maladie correspond au ratio entre les nouveaux cas diagnostiqués pendant une période $(\mathrm{N})$ et les personnesannées vécues dans la population pendant cette période (P). La prévalence d'une maladie est définie comme le nombre de personnes malades à un moment donné $(\mathrm{M})$ par rapport à l'ensemble de la population $(\mathrm{P})$. La durée moyenne d'infection est donnée par le rapport entre $\mathrm{M}$ et $\mathrm{N}$,

7. Cette dynamique se base sur les lois d'une population stationnaire. Si une population, pendant un intervalle de temps (ici entre deux recensements), reçoit et perd un nombre constant d'individus (ici par immigration et émigration), tout en étant isolée d'autres perturbations (ici la mortalité et la natalité), elle peut être qualifiée de stationnaire. La faiblesse du solde naturel et la relative stabilité du nombre d'arrivées entre chaque intervalle intercensitaire (Figure 4) sont en ce sens des signes suggérant une relative stationnarité. 
c'est-à-dire le nombre de personnes infectées à un moment donné divisé par le nombre de nouveaux cas par unité de temps 8 .

$$
\frac{\mathrm{M}}{\mathrm{P}}=\frac{\mathrm{N}}{\mathrm{P}} \cdot \frac{\mathrm{M}}{\mathrm{N}}
$$

L'opérationnalisation de cette formule dans le cas de la migration dans et hors de la ville demande toutefois de définir précisément les termes en présence.

Premièrement, cette formule ne fonctionne que dans le cas d'une nouvelle maladie encore jamais déclarée avant le début de l'observation. Dans notre cas, le nombre de malades étant représenté par le nombre d'immigrés présents en ville, il est nécessaire de soustraire les immigrés déjà présents au moment du recensement précédent pour obtenir $\mathrm{M}$. Par exemple, en 1828, Genève comptait 16087 personnes déclarant une autre origine que Genève. En 1834, ce nombre s'élevait à 18758 . Par conséquent, en 1834, il y avait en ville 2671 étrangers supplémentaires par rapport à 1828, correspondant dans notre formule aux personnes infectées par une nouvelle maladie ${ }^{9}$.

Deuxièmement, le nombre de personnes infectées par une maladie pendant une période donnée se traduit dans le cas de la migration vers la ville par le nombre de personnes étant arrivées en ville. La période considérée est l'intervalle entre deux recensements, de manière à faire coïncider la mesure du flux vers la ville avec les données connues sur les personnes présentes en ville. On observera donc $\mathrm{N}$, le nombre moyen d'arrivées par année pendant l'intervalle intercensitaire (Figure 4).

8. $\quad \mathrm{N}$ étant une fréquence mesurée par l'inverse d'une unité de temps $(t-1)$, cette même unité de temps mesurera donc la durée moyenne d'infection. Ici, en prenant comme unité d'incidence le nombre annuel d'arrivées en ville, on obtient une durée moyenne de séjour mesurée en années.

9. Cette manière de comptabiliser sous-estime légèrement le nombre de nouveaux citadins, dans la mesure où certains des immigrés du premier recensement sont morts ou ont quitté la ville dans l'intervalle. La durée moyenne de séjour calculée est donc légèrement sous-estimée. Puisque le même biais intervient sur chaque période analysée, la comparaison entre les intervalles intercensitaire reste toutefois raisonnablement valide. 
FIGURE 4 Nombre d'arrivées à Genève, 1830-190010

nombre moyen d'arrivées par intervalle intercensitaire

nombre annuel d'arrivées

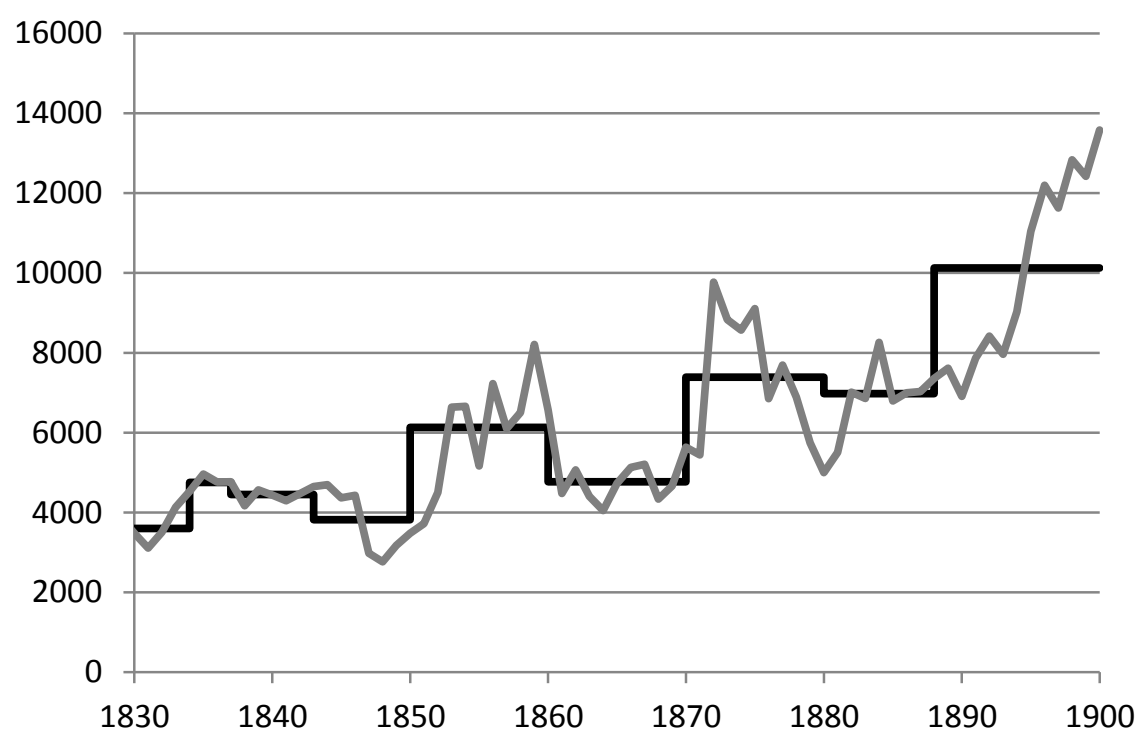

Au final, la durée moyenne de séjour entre deux recensements $\left(\bar{d}_{r}\right)$ s'exprime comme un ratio dont le numérateur est la population étrangère supplémentaire entre le premier recensement $\left(E_{r}\right)$ et le second recensement $\left(E_{r+t}\right)$, et le dénominateur est la moyenne du nombre annuel de permis $\left(P_{i}\right)$ sur l'ensemble de la période allant de $\mathrm{r}$ à $\mathrm{r}+\mathrm{t}^{11}$.

10. En 1844, le nombre de livrets de domestiques est diminué de $2 / 3$ pour tenir compte du fait qu'un nombre important des nouveaux livrets concerne des domestiques déjà présentes auparavant, mais nouvellement concernées par l'obligation de se munir d'un livret. En 1866, le total des permis est diminué d'1/3 (équivalent au total des trois dernières années de livrets de domestiques) afin de tenir compte du report des domestiques dans les permis de séjour.

11. Lorsque le recensement a lieu en janvier ou février (de, et y compris, 1822 à 1843), les permis émis pendant une année de recensement sont comptabilisés avec l'intervalle intercensitaire suivant. Lorsque, au contraire, le recensement a lieu en décembre (dès 1850), les permis émis pendant une année de recensement sont comptabilisés avec l'intervalle se finissant. Les intervalles intercensitaires ( $t$ ) sont calculés comme la différence entre les années de recensements, sauf pour l'intervalle 1843-1850 qui dure une année de plus (de janvier 1843 à décembre 1850). 


$$
\bar{d}_{r}=\frac{M}{N}=\frac{E_{r+t}-E_{r}}{\frac{\sum_{i=r}^{r+t} P_{i}}{t}}
$$

Le calcul de cette durée moyenne de séjour (Figure 5) fait apparaitre une évolution en quatre phases qui semblent coïncider précisément avec les quatre périodes de croissance urbaine distinguées précédemment (Figure 1).

FIGURE 5 Durée moyenne de séjour entre chaque recensement (1830-1900)12

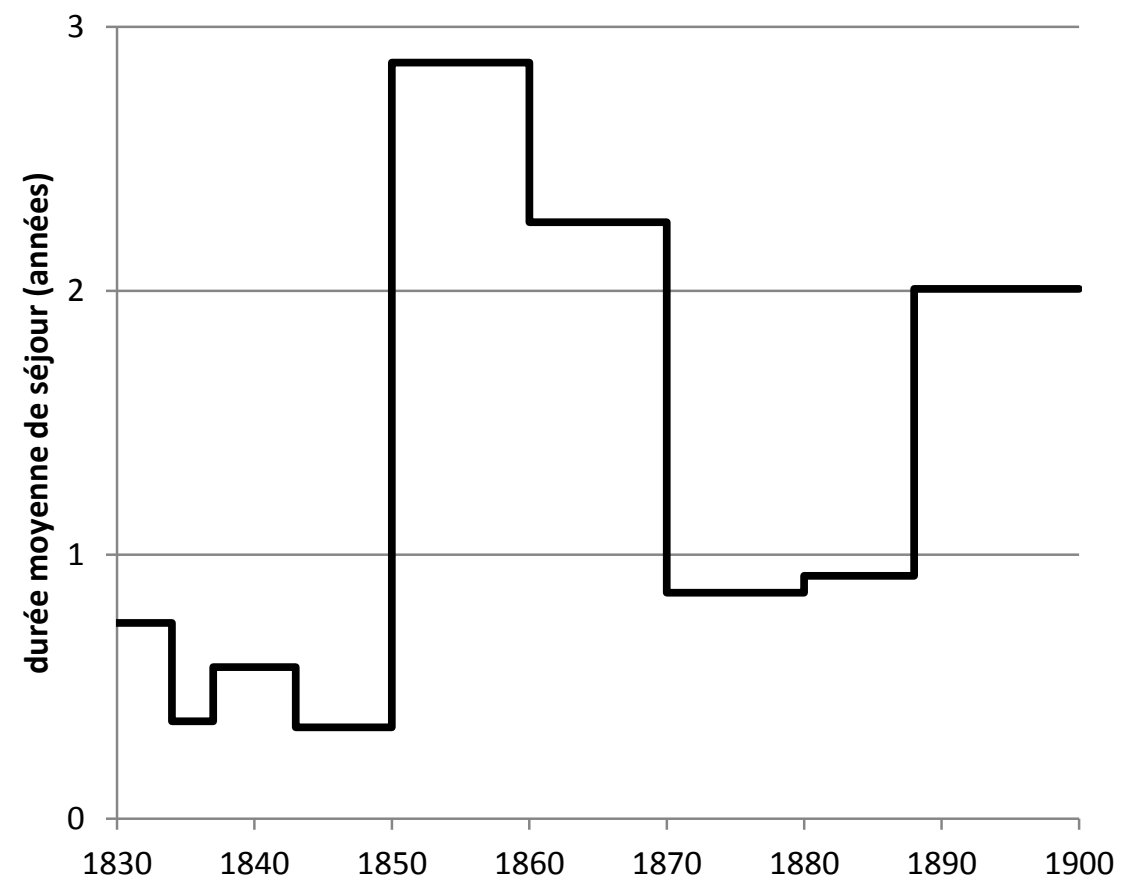

Durant la première moitié du siècle, la durée moyenne de séjour ne dépasse pas une année, mettant en évidence un système dominant de migrations temporaires et/ou saisonnières qui est bien documenté pour Genève (Guichonnet, 1945 ; Perrenoud, 1979 ; Remund, 2012). Puis la durée des épisodes migratoires se fait subitement beaucoup plus lon-

12. La série du nombre de permis délivrés par année ne commence qu'en 1825, rendant impossible l'utilisation des deux périodes intercensitaires 1816-1822 et 18221828. 
gue, atteignant deux à trois ans. Certes, le nombre de nouveaux arrivants s'accroit clairement après 1850, passant environ de 4000 à 6000 permis par an, soit une augmentation de $50 \%$ (Figure 4). Mais la croissance urbaine, elle, augmente d'un facteur de 2,5 dans le même temps (Figure 1). Cette nouvelle dynamique de peuplement débute au moment de la destruction des fortifications et persiste jusqu'au recensement de 1870. Schumacher, dans son étude de la transition démographique de Genève, note d'ailleurs que «le régime démographique genevois se trouve temporairement perturbé entre 1843 et 1870 » et soupçonne déjà «la variable migratoire» d'en être la cause (Schumacher, 2010, p. 318). L'arrivée, après les crises de 1848, d'une nouvelle génération de migrants qui tendent à s'installer en ville avec leur famille et leurs cadres de valeurs offre une explication séduisante à ce ralentissement dans la transition démographique de Genève. Cette nouvelle vague n'implique cependant pas la disparition des migrations saisonnières, les deux formes de migrations ayant probablement coexisté sur une longue période.

Pendant les décennies 1870 et 1880, le nombre d'immigrants continue à progresser, dépassant 7000 par année, alors que la croissance urbaine, elle, retrouve son rythme lent de la première moitié du siècle. Cette évolution désynchronisée de l'afflux migratoire et du rythme de croissance de la ville ne peut être expliquée que par une diminution de la durée moyenne d'installation. Il est possible que cette évolution soit expliquée par un affaiblissement temporaire de la nouvelle vague d'établissements des années 1850-60, faisant réapparaitre plus nettement le flux des migrants temporaires qui avait été masqué pendant un temps. Cette interprétation est compatible avec une hypothèse de Rosental avançant que «la fraction de la population migrante qui était la moins fermement établie était la plus vulnérable aux crises économiques» (Rosental, 2006, p. 471). Or, la période de 1867-69 à 1889-91 correspond à la Longue Dépression, une phase de «quasi-stagnation de l'économie européenne» (Bairoch, 1997, p. 242) qui pourrait bien avoir perturbé les systèmes migratoires pendant deux décennies.

La dernière phase de forte croissance qui commence dans les années 1890 et dure jusqu'à la Première Guerre mondiale n'est que partiellement couverte par les séries des permis qui s'arrêtent en 1900. On note cependant après 1888 une reprise de la dynamique des années 1850 et 1860, qui voit les immigrants se stabiliser à nouveau dans la ville. Combinée à l'augmentation de l'afflux vers Genève, cela explique l'explosion urbaine qu'a connue la ville avant la Première Guerre mondiale. 


\section{Conclusion}

La prise de conscience que les sociétés du passé étaient plus mobiles qu'on ne le soupçonnait n'a pas encore déployé toutes ses conséquences. Certes, les historiens savent maintenant que nos ancêtres se déplaçaient fréquemment et pour des périodes assez courtes. Mais l'évolution à long terme de la durée de ces migrations ainsi que ses conséquences sur le rythme d'urbanisation des villes européennes n'ont pas encore été complètement mesurées. Ces liens entre la durée des épisodes migratoires et la croissance urbaine ont été suggérés depuis un certain temps par les historiens, mais les moyens pour les mesurer restent limités. En utilisant une technique originale, nous avons pu confirmer ce lien de manière empirique.

De concert avec d'autres historiens, nous nous sommes demandés si, réellement «les migrations ont fait grandir les villes» (Jackson, Moch, 1989, p. 31). L'exemple de Genève au 19ème siècle montre que la croissance urbaine est indissociable des deux éléments que sont l'apport migratoire et la stabilisation des migrants. Lorsque l'un des deux faiblit, l'urbanisation ralentit. À l'inverse, les périodes de forte croissance urbaine connaissent à la fois une augmentation des arrivées et une stabilisation des migrants. À Genève, cette conjonction s'observe avant les années 1870 et après 1890, mais pas pendant cet intervalle. Cette évolution irrégulière de la durée des séjours en ville contredit la vision selon laquelle les migrations temporaires disparaitraient progressivement au profit d'une immigration de peuplement. La seconde partie du 19ème siècle montre certes par moment des signes de l'apparition d'une nouvelle dynamique d'installation en ville, mais les migrations temporaires ne disparaissent pas pour autant et reprennent même parfois le dessus.

\section{Références}

AEG BA 9, «Comptes rendus de l'adminstration du Conseil d’État», Archives d'État de Genève.

AEG Étrangers F6-13, "Livrets de domestiques», Archives d'État de Genève.

BAIROCH P. (1997), Victoires et déboirs : histoire économique et sociale du monde du 15ème siècle à nos jours, 2, Les pays développés au 19ème siècle (1815-1914), Gallimard, Paris. 
BAIROCH P., BovÉE J.-P. (1986), Annuaire statistique rétrospectif de Genève, Université de Genève, Genève.

Chesnals J.-C. (1979), "L'effet multiplicatif de la transition démographique», Population (French Edition), 34 (6), pp. 1138-1144.

Engel R., Marin J. (1974), Les émigrés à Genève 1789-1798, Mémoire de licence, Département d'histoire économique, Université de Genève, Genève.

FARON O. (1997), La ville des destins croisés. Recherches sur la société milanaise du 19ème siècle, 1811-1860, École francaise de Rome, diff. de Boccard, Rome et Paris.

GiLle Y. (2009), Genève comme carrefour migratoire au tournant du 20ème siècle : une analyse des registres des permis de séjour et d'établissement suisses et étrangers (1891-1892), Mémoire de maîtrise, Departement d'histoire économique, Université de Genève, Genève.

GUICHONNET P. (1945), "L'émigration saisonnière en Faucigny pendant la première moitié du 19ème siècle (1783-1860)», Revue de géographie alpine, pp. 465-534.

Hatt-Diener M.-N. (2004), Strasbourg et Strasbourgeois à la croisée des chemins. Mobilités urbaines, 1810-1840, Presses universitaires de Strasbourg, Strasbourg.

Henry L., Sauvy A. (1956), Anciennes familles genevoises. Étude démographique: 16ème-20ème siècle, Presses universitaires de France, Paris.

HochSTADT S. (1999), Mobility and Modernity: Migration in Germany, 1820-1989, Ann Arbor, The University of Michigan Press, s.l.

JACKSON J. H., Moch L. P. (1989), «Migration and the Social History of Modern Europe», Historical Methods, 22 (1), pp. 27-36.

LASLETT P. (1977), Family Life and Illicite Love in Earlier Generations, Cambridge University Press, Cambridge.

LORENCEAU R. (2001), Bâle de 1860 à 1920 : croissance et mobilités urbaines, Thèse de doctorat, Université de Tours, Tours.

Magnenat-Luthy C. (1988), L'immigration des Confédérés à Genève en 1910, d'après les permis de séjour et d'établissement suisses, Département d'histoire économique, Université de Genève, Genève.

OrIS M., Perroux O. (2007), «La minorité catholique dans la Rome protestante: contribution à l'histoire démographique de Genève dans la première moitié du 19ème siècle», Histoire des familles, de la démographie et des comportements : en hommage à Jean-Pierre Bardet, J.-P. Poussou, I. Robin-Romero, P. Chaunu (eds), Presses de l'Univ. Paris-Sorbonne, Paris, pp. 201-226.

OrIS M., RITSCHARD G. (2007), «Dynamique socioprofessionnelle dans la Genève du 19ème, enseignements d'une analyse de statistique implicative», 4èmes Rencontres Internationales Analyse Statistique Implicative, R. GRAS, P. ORús, B. PINAUD, P. GREGORI (eds), Castellón de la Plana, pp. 287-300. 
Perrenoud A. (1979), La Population de Genève du 16ème au début du 19ème siècle : étude démographique, Société d'histoire et d'archéologie de Genève, Genève.

Pinol J.-L. (2003), Histoire de l'Europe urbaine, Seuil, Paris.

Preston H. S., Heuveline P., Guillaut M. (2000), Demography. Measuring and Modeling Population Processes, Oxford.

REMUND A. (2009), Les chemins de la migration : analyse de la mobilité étrangère à Genève (1837-43), Mémoire de maîtrise, Département d’histoire économique, Université de Genève, Genève.

REMUND A. (à paraître), «Rester ou repartir? Une analyse des usages de la ville par les migrants dans la Genève des années 1837-1843», Annales de Démographie Historique, (2).

Rosental P.-A. (2006), «Between Macro and Micro: Theorizing Agency in 19th-Century French Migrations», French Historical Studies, 29 (3), pp. 457-481.

Rosental P.-A. (1999), Les sentiers invisibles, espaces, familles et migrations dans la France du 19ème siècle, Paris.

SCHUMACHER R. (2010), Structures et comportements en transition : la reproduction démographique à Genève au 19ème siècle, Peter Lang, Bern.

ZeuInsky W. (1971), "The Hypothesis of the Mobility Transition», Geographical Review, 61 (2), pp. 219-249. 